\title{
Foreword to the Special Focus on Mathematics and Algorithms for CAM and CNC
}

\author{
Hongbo Li · Rida T. Farouki · Dingkang Wang
}

(C) Springer Basel AG 2012

The fast development of advanced manufacturing technology has witnessed the growing importance of mathematical methods and algorithms, ranging from algebraic geometry, discrete geometry and differential geometry to differential equations, computational mathematics and computer mathematics. Conversely, problems arising from the field of advanced manufacturing have also stimulated the development of such branches in pure and applied mathematics as computational geometry and mathematics mechanization.

Mathematics and Algorithms for Computer-Aided Manufacturing, Engineering and Numerical Control (MAMENC) is a new forum focusing on the interaction between the side of mathematical methods and algorithms, and the other side of computer-aided manufacturing (CAM), computer-aided engineering (CAE) and computer numerical control (CNC). MAMENC 2011 was organized by the National Center for Mathematics and Interdisciplinary Sciences of China (NCMIS), and held in Beijing on October 24-26, 2011.

This special focus is a selection of research papers from the submissions to MAMENC 2011. All these papers contain original work not published elsewhere and are refereed according to the standards of Mathematics in Computer Science (MCS). Topics of interest for the special focus include: tool path planning and collision avoidance, and time-optimal interpolation for $\mathrm{CNC}$ controllers. There are five papers in this special focus. The first three papers deal with tool path planning and collision avoidance, and the rest two deal with CNC interpolation. Below we give a brief introduction to their contents.

The paper by Alessandro Gasparetto, Paolo Boscariol, Albano Lanzutti, and Renato Vidoni addresses the general problem of trajectory planning in robotics, with a nice review of the robotics literature and a discussion on finding an optimal trajectory for a given path.

The paper by Hongbo Li, Shoubin Yao, Ge Li, Yuanjie Liu, and Lixian Zhang gives explicit power series solutions in symbolic form to the problem of generating tool paths of a ball-end cutter on a free-form surface, under the initial condition that the first tool path is given.

H. Li $(\varangle) \cdot$ D. Wang $(\varangle)$

KLMM, AMSS \& NCMIS, Chinese Academy of Sciences, Beijing 100190, China

e-mail: hli@mmrc.iss.ac.cn

D. Wang $(\varangle)$

e-mail: dwang@mmrc.iss.ac.cn

R.T. Farouki $(\varangle)$

Department of Mechanical and Aeronautical Engineering,

University of California, Davis, CA 95616-5294, USA

e-mail: farouki@ucdavis.edu 
The paper by Stephen P. Radzevich outlines a novel method for optimal sculptured surface generation, the so-called DG/K-based approach (Differential Geometry of surfaces, and Kinematics of multi-parametric motion of a rigid body).

The paper by Yongqing Wang, Haibo Liu, and Sennan Yu proposes a NURBS surface interpolation algorithm. In the real-time interpolation stage, global flexible feedrate control and local adaptive acceleration/deceleration optimization strategies are available.

The paper by Hongbo Li, Xiaoshan Gao, Lixian Zhang, and Ruiyong Sun gives a real-time interpolation method for G01 codes without resorting to smooth curve approximation. The method is based on discrete geometry of the piecewise linear trajectory defined by the G-codes.

We thank the authors, the numerous referees, and the managing editor of MCS, Dongming Wang, for their great support. 